Draft of Chapter that will appear in In E. Dupoux (Ed.).(2001). Essay in Honor of Jacques Mehler. Cambridge, MA: MIT Press.

Counting in Animals and Humans

Rochel Gelman and Sara Cordes

UCLA and Rutgers*

* Corresponding Authors' address: Psych \& Cognitive Science, RuCCS, 152 Freylinghuysen Road, Rutgers Univ, Piscataway, NJ 08854--8020: rgelman @ ruccs.rutgers.edu 
Gelman \& Cordes

I. On Phylogenetic Continuity and Possible Ontogenetic Implications

Throughout the century many anthropologists, psychologists, and historians of

mathematics have assumed that there is a sharp discontinuity between the arithmetic abilities of animals, infants, young children and early or "primitive" societies, on the one hand and, older children and acculturated societies, on the other hand. Over and over again reports of birds, dogs, apes, bees, etc., keeping track of the number of items in small collections are ascribed to a "number sense faculty"1 (e.g., Dantzig, 1967; McCleish, 1991; Ifrah 1985). Animals' seeming failure to work with larger set sizes was taken as evidence that they could not count. Indeed, the ability to count was considered too abstract a capacity for animals. "They never conceive absolute quantities because they lack the faculty of abstraction" (Ifrah, p. 4, 1985).

The contrast between a perceptual, non-quantitative mechanism for enumerating small sets of items (i.e. 3 -5) and a 'true' quantitative mechanism for representing larger numbers also is fundamental to ontogenetic and/or cultural discontinuity theories (e.g., Baroody, 1992; Fuson, 1988; Fischer, 1992; Piaget, 1952; McCleish, 1991; Sophian, 1995). The Mehler and Bever (1967) demonstration that 2-year-olds succeeded on a modified version of Piaget's famous number conservation task has been downplayed on the grounds that rather small set sizes (4 - 6) were used. A similar argument is used with respect to findings from Gelman's magic task, which was comprised of a 2-phase procedure. During Phase 1 expectancies for two N's were established; Phase 2 followed after a surreptitious transformation occurred (e.g, Bullock \& Gelman, 1977; Gelman, 1972; Gelman \& Gallistel, 1978) A combination of children's surprise levels, choices, and verbal explanations across the two phases of the experiment revealed early knowledge of a number-invariance scheme. These young children treated surreptitous changes in length,

\footnotetext{
1. The meaning of the phrase "number sense faculty" is used here as it was by the cited writers. In modern psychological writings, its meaning converges with the use of "subitizing" when it is meant to refer to a non-numerical perceptual processing mechanism. It should not be confused with Dehaene's (1997) book title Number Sense which is about a wide range of numerical cognitions, their origins and possible brain localizations.
} 
Gelman \& Cordes

density, item kind, and color as irrelevant to the expected number(s). In contrast, they inferred that addition or subtraction had to have occurred when they encountered unexpected changes in number(s). Even 2-1/2 year-olds used number ordering relations, that is, they could pair the expectancy phase values of 1 vs 2 with the unexpected values of 3 vs 4 . Even though the children in the various magic experiments often counted and explained their decisions, these results frequently are attributed to a perceptual number apprehension device, or "subitizing".

When "subitizing" is used as above, it typically is taken to mean that "twoness" and "threeness" are like "cowness" and "treeness", that is, that there are unique percepts for each set of small numbers. Another way of putting the claim is to predict that the reaction time function in the small number range is flat; that is, it should take the same amount of time to identify the quality "twoness" as it does to identify "threeness" and "fourness". An alternative interpretation of subitizing allows that the items are individuated in the enumeration process. In fact, there are increases in reaction time as a function of set size, even within the small number range (Balakrishnan \& Ashby, 1992; Folk, 1988; Klahr \& Wallace, 1975; Trick \& Pylyshyn, 1993; see Dehaene, 1997, Gallistel \& Gelman, 1992, for reviews).

Proposals that infants use object files (Carey, this volume; Simon, 1999) represent an alternative way to put the assumption that there is a limit on the number of items that can be processed at a given time, and that small sets of $\mathrm{N}$ are not processed in a truly numerical way. They are favored because they provide an account of how items are individuated within the small number range. Since there is a limit to the number of object files that can be opened at one time (Trick \& Pylyshyn, 1993), it is not surprising that authors who endorse the object file account (e.g., Carey, this volume) favor a discontinuity account of how small and larger numbers are processed.

Some discontinuity arguments are coupled with the idea that the ability to engage in numerical cognition depends on acculturation and the development of abstract reasoning 
Gelman \& Cordes

structures. There are at least four reasons to challenge a strong phylogenetic discontinuity hypothesis. First, one need not assume that an abstract classification scheme mediates the ability to count. Second, there is an ever-growing body of findings that animals do count nonverbally, well beyond numbers in the range of $2-5$ or 6 . Third, there is evidence that adult humans share a nonverbal counting mechanism with animals, one that operates in both the small and large number ranges (see below, as well as Cordes, Gelman, Gallistel \& Whalen, under review). Fourth, the set of principles governing the mechanism that generates nonverbal cardinal representations, or what Gelman and Gallistel (1978) dubbed cardinal numerons, embeds an effective procedure for determining a successor.

What about the matter of phylogenetic continuity? As we shall see, there is evidence that adult humans can use the same nonverbal counting mechanism when working in either the small or large number range. As we shall see, this is relevant to the interpretation of infant number data. Similarly, our findings that there are nonverbal generative processes for achieving representations of discrete (as opposed to continuous) quantities are relevant to an account of the differential ease with which humans come to learn verbal and other symbolic systems and why humans find it easier to re-represent natural (counting) numbers as opposed to rational and other kinds of real numbers.

\section{On The Nature of Natural Numbers}

There are extensive treatises on the nature of number and it is not our intent to provide an exhaustive review. Our goal is to bring to the fore three lines of theoretical reasoning about the psychological nature and or generation of counting numbers. The first two are more "domain-general" than not. That is, they constitute efforts to build up the ability to generate and reason with natural numbers from non-numerical primitives. Of these two, one is rooted in the assumption that a given count or number represents an abstract class or set. The second, although not independent of the first, places considerable emphasis on the role of language. Neither of these accounts is applied to animal data. The third account is domain-specific and it assumes that, counting principles and its 
Gelman \& Cordes

representations, in combination with arithmetic reasoning principles, constitute a foundational domain unto themselves. It is applied to accounts of nonverbal counting and arithmetic reasoning, be this in animals, preverbal children, or groups who use nonverbal counting systems.

The Classification and Set-Theoretic Approach.

This view is very much related to traditional theories of concept acquisition and set theoretic definitions of numbers. From the developmental perspective, the idea is that, at first, children cannot even classify like items with identical properties (e.g., Piaget, 1952; Vygotsky, 1962; Werner, 1954). Then, they move on to classifying together items that are perceptually identical, then items that differ in a property but share a shape, and so on, until items that differ considerably with respect to their surface properties are treated as members of an abstract class, 'things.' It is assumed that children have to develop classification structures in order to classify at this level of abstraction. In the case of numbers, the abstract capacity to count is related to the idea that a given number, say 5 , represents, the set of all sets of that number, be these sets of 5 dogs, 5 ideas, 5 things in a room, or Lewis Carroll's miscellaneous collection of five kinds of things: ships, shoes, sealing wax, cabbage and kings. Movement from the concrete to the abstract takes a very long time, even for man. Sir Bertrand Russell, who made fundamental contributions to the settheoretic approach to number, put it thusly: "It must have required many ages to discover that a brace of pheasants and a couple of days were both instances of the number two" (Dantzig, p.6, 1967). Further discussion of the set-theoretic approach is presented in Chapter 11 of Gelman and Gallistel (1978).

The Language Dependency Approach.

There are at least two kinds of language-dependency arguments. One treats language as a necessary prerequisite. In this case there can be no such thing as preverbal or nonverbal counting. Further, understanding of the meaning of the count numbers, as well as their arithmetic use, emerges from inductions based on the use of counting words in the 
Gelman \& Cordes

context of subitizing, and rote counting experiences. Fuson's (1988) treatment of verbal counting is a possible example of this account. So too is McLeish's (1991), who wrote: “The reason for animals' inability to separate numbers from the concrete situation is that they are unable to think in the abstract at all - and even if they could, they have no language capable of communicating, or absorbing, such abstract ideas as 'six', or a 'herd'" (p.7).

A second kind of language-dependent argument allows that there is, or could be, a system for nonverbal counting and/or arithmetic that does not generate discrete values. However, this system is seen as both separate from and discontinuous with a verbal counting system. Carey (this volume) develops one form of such an account but there are others (e.g., Bloom, 2000; Butterford, 1999; Dehaene, 1997). The verbal counting system usually is treated as being closely related to and emergent from the semantic/syntactic linguistic system of quantifiers. If the ability to count is indeed dependent on the development of language, then animals, who are nonlinguistic, and infants, who are prelinguistic, definitely cannot really count.

The Domain-Specific Approach

We favor a domain-specific approach to the count numbers and their arithmetic function and define a domain of knowledge in much the same way that formalists do, by appeal to the notion of a set of interrelated principles. A given set of principles, the rules of their application, and the entities to which they apply together constitute a domain. Since different structures are defined by different sets of principles, we can say that a body of knowledge constitutes a domain of knowledge to the extent that we can show that a set of interrelated principles organize the entities and related knowledge as well as the rules of operation on these. Counting is part of a number-specific domain, because the representatives of numerosity (what we call numerons) generated by counting are operated on by mechanisms informed by, or obedient to, arithmetic principles. For counting to provide the input for arithmetic reasoning, the principles governing counting must complement the principles governing arithmetic reasoning. For example, the counting 
Gelman \& Cordes

principles must be such that sets assigned the same numeron are in fact numerically equal and the set assigned a greater numeron is more numerous than a set assigned a lesser numeron. The counting principles and their relation to the arithmetic operations of addition, subtraction, and ordering are illustrated in Figure 1.

Insert Figure 1 About Here

Note that there is nothing in this formulation that yokes counting to the use of number words or other numerical symbols. Indeed, this is why we introduced terms to distinguish between nonverbal and verbal or symbolic representations: numerons and numerlogs (Gelman \& Gallistel, 1978). As long as the generative process honors the oneone, stable and cardinal principles, and tolerates a wide range of item types, we can say that it is a candidate counting device. A pulse generator and a computer are examples of such entities.

Nor is it necessary to assume that a complex hierarchization scheme mediates the ability to come to understand that one can count a heterogeneous collection if one simply thinks of them as 'things'. Under these circumstances, the primary requirement is thingness or individuation of the members of the to-be-counted set. That is, the only requirement is that each thing can be treated as separable one from each other, either physically or mentally. Often it is the case that things being counted are separably moveable items, processes that serve figure-ground abilities can help us sort things from nonthings. Similarly, perceptual tendencies to separate auditory events (tones or speech units) can help one keep track of already-counted vs. to be-counted inputs. Of course, there is much work to do on the question of what we will tolerate as a countable part, whether young children will count the Lewis Carroll set as five, and so on (Shipley \& Shepperson, 1990). But the research agenda as to what counts as a separate countable is a 
Gelman \& Cordes

different one than one that ties counting prowess to the development of the ability to use hierarchical classification systems.

In the next section we present our model of this counting device, namely that it is just the machinery that generates the real numbers that represent countable, ordered, quantities, that is the cardinal numerons. (Gallistel \& Gelman, 1992; Gallistel, Gelman \& Cordes, 2000).

\section{On Nonverbal Counting and the Accumulator Model}

We favor a direct descendant of the Meck and Church (1983) accumulator model for animal counting data. As shown in Figure 2, the model uses a discrete process to generate ordered quantitative representations, for both animals and humans (Gallistel \& Gelman, 1992; Whalen, Gallistel \& Gelman, 1999). In addition, it is assumed that humans build bi-directional mappings between the quantities generated by the counting processes and the various count words and symbols that stand for a given cardinal value. To expand on the nature of the model, we begin by reviewing some animal counting data. Then, we turn to evidence that humans share the nonverbal counting mechanism. To end, we return to consider the idea that there are small number mechanisms that are independent of counting, be these perceptual or object file.

\section{Insert Figure 2 About Here}

\section{Evidence that Animals Count Nonverbally.}

As it turns out, there is a set of experiments showing a variety of animals possess some surprising numerical abilities. This includes the ability to reason and count about rather large set sizes. Of course, whatever the process by which animals do count, it surely is a nonverbal one. Brannon and Terrace (2000) showed that monkeys can order stimuli that display as many as 9 items. Once trained to arrange sets of 1,2,3, and 4 items in 
Gelman \& Cordes

ascending order, they spontaneously generalized to novel sets of items containing 5-9 items. Platt and Johnson's (1971) study of the ability of rats to count is an especially nice example of nonverbal counting in animals. Their data are shown in Figure 3.

Insert Figure 3 About Here

Platt and Johnson's rats had to press a lever an experimenter- determined number of times in order to arm a feeder. The target values of $\mathrm{N}$ varied from 4 to 24 . If a rat pressed too few times, the counter that was keeping track of the number of presses was reset, meaning that the rat had to start his count anew. The rats' responses were systematically controlled by the target value of the requisite number of presses in a given block of trials. As the target value increased for a block of trials, so did the modal number of presses. In addition, set size increases led to a systematic effect on the animal's accuracy, that is, the increases so does the mean number of responses width of the distribution was proportional to the mean number of responses for a given experimental N. As a result, the outcome of dividing the standard deviation by the mean was a flat function. That is, the data from the experiment can be said to exhibit scalar variability. This characterization of the rat's counting behavior is common to a number of other studies with animals and is well-modeled by the nonverbal counting mechanism shown in Figure 2.

In our model of nonverbal counting (Gelman \& Gallistel, 1978; Gallistel \& Gelman, 2000; Gallistel, Gelman, \& Cordes, in press) cardinal numerons are represented by magnitudes generated by an accumulator process. As shown in Figure 2, each unit enumerated is represented by an additional increment in the accumulator, analogous to pouring a fixed amount of liquid into a beaker. The cardinal value of the counted set of items or events is represented by the final magnitude in the accumulator (analogous to the total amount of liquid in the beaker). As the magnitude in memory increases, there is a proportional increase in the variability of the magnitudes read from that memory--in the trial 
Gelman \& Cordes

to trial variability of the magnitudes actually remembered. The proportional variability in the magnitudes read from memory leads to scalar variability in the behavioral data, because the processes that translate remembered numerical magnitudes into numerically governed behavior make ratio comparisons between remembered (target) and current estimates of numerosity. These and other findings imply that a system of simple arithmetic reasoning (addition, subtraction, ordering) mediates the translation of numerical magnitude into observable behavior (see Gallistel, Gelman, \& Cordes, in press, for evidence that the reasoning processes also include multiplication and division).

Although the product of the accumulator processes is a magnitude, its processes are discrete and embody the counting principles. That is, the counting processes are governed by the how-to counting principles, these being the (1) One- one principle: There is one and only one discrete pour (or, if you prefer, pulse) for every item, and as many pours as there are items to count; (2) Ordering principle: The process is a sequential one, generating one and only one quantity at a time; and the (3) Cardinal principle: The last magnitude generated by this discrete process has a special status -- it is written to memory and represents the cardinal value of the counted set. Finally, the memory is variable -- this is indicated by the sloshing line in the figure. Since it is a discrete process that renders successive quantities, there is a perfectly clear sense in which one gets the next magnitude. Given that there is a next relation that holds among the quantities, it follows that these represent discrete quantity, i.e., countable quantity. This sets the stage for our idea that humans come to map nonverbal quantity representations generated by counting to verbal and/or other symbolic representations of the natural numbers (see the bottom of Figure 2). Several lines of evidence are consistent with this proposal. In what follows we focus on the evidence that adults use a nonverbal counting mechanism that shares the key properties of the animal one presented above. The final section of the chapter returns to the mapping proposal. 
Gelman \& Cordes

Adults Also Count Nonverbally.

It has been known at least since the work of Moyer and Landauer (1967) that the mean reaction latency for adult human judgments of the numerical order of numerals increases with relative numerical proximity: the smaller the percent difference, the longer the mean latency (Dehaene, 1990; Holyoak, 1978, 1982; Moyer, 1973). The increase in reaction latency suggests that adult human judgments of numerical order are based on an underlying magnitude representation of numerosity. Whalen, Gallistel and Gelman (1999) have presented evidence that adult humans can use a nonverbal counting mechanism to generate magnitudes. In a key press task, adults were first shown an Arabic numeral and asked to rapidly press a key, without counting, until they felt they had arrived at the represented number. The number of key presses increased linearly with target number and so did the trial-to-trial variability in the number of presses for a given target number. On a different task, subjects saw a rapidly, but arhythmically, flashing dot and indicated how many flashes they felt they had seen, without counting. Similar results to the key press task were obtained, providing further support for nonverbal numerical competence in adult humans similar to the one observed in nonverbal animals.

More recently Cordes, Gelman, Gallistel and Whalen (under review) asked adult subjects to talk while doing the nonverbal counting task. Depending on the condition, subjects either recited "Mary had a little lamb" or the word "the", over and over again, while pressing a key as fast as they could as many times to represent the value of target Arabic number. We reasoned that these talk-aloud conditions would be an effective way of preventing verbal counting, sub-vocal or otherwise, because you have to say something just at the moment when you would say the count word if you were counting verbally.

Again, we obtained the signature pattern of scalar variability. Together the above results support both of our proposals about human counting: we have available a nonverbal counting mechanism and engage in a bi-directional mapping process. The latter allows them 
Gelman \& Cordes

to map either from a given numeric symbol to the nonverbal counting of taps or the nonverbal counting of flashes to numeric symbols.

At first blush, the idea that adults use any nonverbal counting mechanisms to deal with any arithmetic tasks, let alone one that has scalar variance, might seem decidedly odd. After all, the great advantage of the language of mathematics is that it makes things precise and exact. But ponder the fact that it takes longer for people to judge that $99>98$ than it does to judge that $4>3$, even though the differences are exactly equal. Similarly, it takes people longer to judge that $9>8$ than it does to judge that $9>4$. These size and distance effects are further evidence that adult humans can and do use a nonverbal counting mechanism that exhibits scalar variability. Still, the fact that we also know that, mathematically, (99-98) $=(4-3)$ makes it obvious that there is more than one way we can represent, define and reason about numbers (See also Dehaene, Spelke, Pinel, Stanescu, Tsivkin, 1999; Droz, 1992).

Discontinuity Between Nonverbal and Verbal Counting?

As indicated at the start of this essay, the notion of a non-numerical mechanism, be it a perceptual apprehension or an object-file one, is contrasted with the ability to count and reason about abstract representations of number and quantity. The distinction is grounded in one or both of two different kinds of accounts. First, there is the presumption that there is either a non-numerical, or at best, a quasi-numerical mechanism for processing displays that contain small set sizes, about 3-5. This proposal is a common feature of histories of mathematics or the anthropology of "early man". It also has support from a number of developmental and cognitive psychologists. Many who study the abilities of infants, young children, and adults to discriminate between different set sizes also favor the hypothesis that a non-numerical mechanism accounts for the limited ability of infants to discriminate between 2 and 3 but not larger set sizes, young children's difficulties counting verbally with set sizes that go beyond 3 or 4 , and the fact that children and adults respond more rapidly to sets in the small number range as opposed to ones larger than 6 or 7 . 
Gelman \& Cordes

Whatever the details of these accounts, they all share a discontinuity assumption. This is that the nonverbal representation of the small numbers is fundamentally different from the nonverbal representation of the bigger numbers.

If small numerosities were represented by something like sets of tally marks, say, object files, and larger numerosities by magnitudes (Carey, this volume; Uller, Carey, Huntley-Fenner \& Klatt, 1999), then these two modes of representation would seem to be like oil and water. How does one subtract a set of two tally marks from a magnitude representing, say, numerosity 8 ? By what mechanism does the brain carry out binary combinatorial operations that span the two ranges? If small numerosities were represented by something like a perceptual classification process, say where "twoness" and "threeness" are like "cowness" and "treeness" and numerosities greater than 4 were represented by quantities, the combinatorial still exists. Indeed, it gets worse since there is nothing we know about perceptual classification processes that leads to an ordering rule such that cowness somehow represents more than treeness. Nor is it clear how one would perform operations of addition and subtraction. Although it is possible to order small sets of tally marks and even add or subtract them, there is the problem that the operation of addition would not be closed, that is, the addition of "3" and "3" would be impossible because the value "6" is not included in the small number system. Further, unto themselves, object files do not generate a "next" entry. For this to happen, at least the operation of addition has to be added to the number processing repertoire. We do not mean to deny that infants use object files to individuate items. We do deny that, on their own, they lack intrinsic quantitative properties (Simon, 1999).

Returning to Figure 3, we draw attention to two characteristics of the data pattern. First, the animals made errors on some trials in the small number range. Second, there is no indication that there is a change in the statistical functions describing performance across the full range of the numbers used in the experiment. That is, there is no clear reason to hold that performance on the small $\mathrm{N}$ of 4 was governed by a different process than 
Gelman \& Cordes

performance on the larger set size values. This consideration led Cordes et al. (under revision) to include numerals representing values in the small, "number sense" range $(2,3$, $4,5)$ as well as ones assumed to be in the nonverbal counting range $(8,13,20 \& 32)$. Each of 4 subjects were run 20 trials on every one of the set sizes. As already indicated above, these 'talking' while nonverbal counting data are characterized by scalar variability. This means that all subjects made mistakes, even when given the target values of 2, 3, and 4. Note that the shapes of the relevant distributions shown in Figure 4 are very much like those in Figure 3, the latter being from data collected with rats. Moreover, as reported in Cordes et al., the coefficients of variation for targets "2", "3", "4", and "5" do not differ from those beyond that range. So, the variability in mapping the nonverbal counting system to a graphemically specified target number is scalar all the way down.

\section{Insert Figure 4 about Here}

The probability that adults in the Cordes et al. study made an error when working with small N's was small. Had we not used a large number of trials, we might have missed the systematic tendency to err even in this range. This does not mitigate the fact that the function describing the relationship between number of responses and errors is the same in the small and larger number range. Given that animals and adults err when generating a nonverbal representation of small values of number, there is a straightforward design implication for studies with young children that uses a variant of a discrimination paradigm. Given that the underlying variance of the memory for a given quantity representation increases systematically as does set size, the probability of $\mathrm{X}$ number of children succeeding decreases as a function of N. Put differently, the probability of making a memory error when comparing the values in question will increase as does $\mathrm{N}$. As a result, the fact that a given $\mathrm{N}$ of infants pass discrimination or habituation tasks with small, but not somewhat larger numbers, could well be due to a statistical artifact as 
Gelman \& Cordes

opposed to a common underlying competence mechanism that deals both with N's outside and inside of the "subitizing range". In order to protect against this possibility, it is important to find ways to run repeated trials with infants and young children. Since this is not an easy task to accomplish, investigators should at least increase the number of participants. Studies which incorporate these design features are needed in order to choose between a position like ours, that assumes there is a continuity between the ability of animals and humans to engage in nonverbal counting, and ones that favor a variant of a discontinuity hypothesis.

The Cordes et al. results bear on another issue raised by Carey (this volume). She states that "analog magnitude representational systems do not have the power to represent natural number" (p. x). This is not so. The natural numbers are a special subset of the real numbers. Since our model provides an effective procedure for determining a nonverbal successor, it encompasses a foundational feature of the natural numbers. This issue illustrates the importance of the question of how the implicit becomes explicit and whether or not the implicit system underlies learning about the re-representational tools, be these linguistic or alternative symbolic systems.

\section{On Learning About Verbal and Other Symbolic Re-Representations of Number}

The availability of a nonverbal arithmetic-counting structure facilitates the verbal counting system. This is because learning about novel data is aided whenever it can take advantage of an existing mental structure. The learner is more likely to select together those pieces of data that the culture offers as relevant. When the use-rules organizing the sequential sounds of a culture's particular count list honor the counting principles, then they can be mapped to the available, implicit list. In this case, young learners have a way to identify inputs that can be mapped to the counting principles and then the principles can begin to render the lists numerically meaningful. Preverbal counting principles provide a conceptual framework for helping beginning language learners identify and render intelligible the individual tags that are 
Gelman \& Cordes

part of a list that is initially meaningless. It also provides a structure within which they build an understanding of the words (Gelman, 1993).

Of course, it is one thing to master a culture's language rules that makes it possible to generate successive next numerals, forever. The issues of early variability are complex (Gelman \& Greeno, 1989; Gelman 1993). Here we focus on ones related to the topic of continuity and discontinuity. First, even adults, unlike computers, are less than enthusiastic about memorizing long lists of sounds that are not intrinsically organized. There is nothing about the sound "two", that predicts the next sound will be "three", or the sound "three", that predicts the next sound will be "four", and so on. This is a non-mathematical, information processing reason to expect the task of committing even the first nine count words to take a considerable amount of time, and it does — as much as 2-4 years (Miller, Smith, Zhu, \& Zhang, 1995). Since the nonverbal counting process allows for the generation of a successor for each representation, there is yet a further serial learning problem the child has to confront. This is the mastery of the structure of the base-rules embedded in a particular language's counting system.

The English count list lacks a transparent decade rule for at least the first 40 entries, and probably, first 130 count words. Learning this rule ends up taking a surprisingly long time. Hartnett (1991) found that a number of Kindergarten and Grade 1 students had yet to catch on to the procedure for generating the count words in the hundreds (Hartnett, 1991), most probably because many English speaking children think one hundred is the next decade word after ninety. If so, in order for them to induce the 100's rules in English, they need to encounter relevant examples. Cross-cultural findings are consistent with this argument. Chinese has a more transparent base-10 count rule for generating subsequent count words, even for the tens and decades. Although the rate at which English and Chinese speaking children learn the first nine count words is comparable, Chinese speaking children learn the subsequent entries at a much faster rate (Miller et al., 1995; Miller \& Stigler, 1988). Cultural variables also influence the extent to which limited count lists are used. The first author well recalls a conversation with an Ethiopian man who had moved to Israel. He was readily able to provide 
Gelman \& Cordes

the words in Falshel for count words well into the thousands. However, when we asked him to name the word for $1,000,000$, he used the Hebrew word. We pointed this out and he responded "there is nothing we counted to 1,000,000 when I lived there." Other examples of the rapid assimilation of well-formed generative count lists as soon as a culture encounters them are provided in Crump (1990), Gvozdanoviâc (1999), and Zaslavsky (1973)

A culture's rapid uptake of a count list that is better than one already in use is especially interesting. For us, these are examples of minds in search of the kind of list that best serves counting principles. It is as if the minds of people in these cultures are already prepared to adopt counting tools that will allow them to generate verbal counting successors. A related phenomena was reported by Hartnett and Gelman (1998). They engaged children in Kindergarten as well as Grades 1 and 2 in a thought experiment designed to encourage them to reach an explicit induction of the successor principle. To do this they asked children to ponder a larger number, then add 1 to that, then again add 1, and so on. Periodically, a child was asked if the number she was thinking about was the largest there could be or whether there were more numbers. Then, depending on the child's answer they were asked what the biggest number was and whether they could add to it or what was the next. A very large percentage of the children caught on to the successor principle, indeed, they went around telling their friends about their new knowledge.

The majority of children in the Hartnett and Gelman (1998) study listed numbers at least as great as 100 when first asked what they thought was a big number. Some however, said numbers well below 100, e.g., 29. These children resisted the idea that there is always a larger or next number. Still, it would be premature to conclude that these younger children lacked knowledge of the successor principle. They did know that there would be yet another, and another, and another, and ......... another dot --- given a dot-generating machine and an endless supply of paper. It therefore is more likely one needs to know the rules for making up the next verbal number. Indeed, one child said "you can't do that, unless someone makes up a new count word". These are all considerations about the kinds of explicit knowledge that 
Gelman \& Cordes

develop, and not whether the successor principle is implicitly available. This highlights why it is critical to keep separate the processes which generate the representation and the nature of that representation.

Differences in the structure of the linguistic and/or symbolic entities that notate different kinds of numbers are related to the question of whether the learning about them is facilitated by existing nonverbal mathematical representations or not. Knowledge of the counting principles can facilitate learning how to interpret the words and marks for the integer. They are discrete and are used in a way that maps directly to the counting principles. Most importantly, they map to the unique mean values that the nonverbal system generates with the counting processs. Note that there is no corresponding word for the variance associated with the mean. Hence, the verbal instantiation of the counting procedure highlights what are the mathematical features of the system: it generates discrete, successively, ordered values. This is part of our account of how it can be that people can interpret the count words at two different levels: (a) as a map to its nonverbal representation and hence as a quantity; (b) as a mathematical entity that has a precise mathematical meaning.

Although count words map in a straightforward way to discrete nonverbal entities, i.e., the means of the distributions generated by application of the nonverbal counting process, the same is not true for rational numbers or any other real numbers. Worse yet, the mathematical principles underlying the numberhood of fractions and decimals are not consistent with the verbally instantiated principles of counting. In fact, the verbal/notational system that children master for counting numbers is inconsistent with that for fractions and decimals. One cannot count things to generate a fraction. Formally, a fraction is defined as the division of one cardinal number by another: This definition solves the problem that there is a lack of closure of the integers under division. To complicate matters, some counting number principles do not apply to fractions. Rational numbers do not have unique successors; there is an infinite number of numbers between any two 
Gelman \& Cordes

rational numbers. So, one cannot use counting based algorithms for ordering fractions, for example, $1 / 4$ is not more than $1 / 2$. Neither the nonverbal nor the verbal counting principles map to a tripartite symbolic representation of fractions - two cardinal numbers $\mathrm{X}$ and $\mathrm{Y}$ separated by a line - whereas the formal definition of a fraction does.

Hartnett and Gelman (1998) reasoned that if children bring to their early school mathematics lessons the idea that the language of mathematics is grounded in counting principles and related rules of addition and subtraction, their constructivist tendencies could lead them to distort fraction inputs to fit their counting based number theory. These authors reasoned that, therefore, early verbal knowledge of number might serve as a barrier to learning about the meaning and notational system for fractions. In contrast, they expected that children of the same age would have a relatively easy time engaging in a thought experiment designed to encourage the explicit induction of the successor principle, here because the structure of the tobe-learned inputs mapped to their existing arithmetic-counting structure. These predictions were supported. The children in the Hartnett and Gelman (1998) study were in Kindergarten through Grade 3, rather young in school age. However, even college students persist in treating rational numbers as if they were odd variants of natural numbers. For example, students in a Chemistry class at UCLA said they could not graph a particular problem "because their graph paper did not have enough squares".

The Hartnett and Gelman findings highlight the need to pay attention to the extent to which the nonverbal representations of numbers map readily to the re-represented verbal and notational systems of number. The absence of an easy map between the nonverbal representations of rational and other real numbers and verbal or symbolic representations of these numbers is a serious impediment to learning the cultural creations for representing such numbers. 
Gelman \& Cordes

\section{References}

Baroody, A. J. The development of preschoolers' counting skills and principles. In J. Bideau, C. Mejac, \& J-P. Fischer (Eds.). Pathways to number: Children's developing numerical abilities. Hillsdale, NJ: Lawrence Erlbaum Associates, (pp. 99-126).

Bloom, P. (2000). How children learn the meanings of words. Cambridge, MA.: MIT Press.

Brannon, E. M., \& Terrace, H. S. (1998). Ordering of the numerosities 1 to 9 by monkeys. Science, $\underline{282}, 246-9$.

Bullock, M., \& Gelman, R. (1977). Numerical reasoning in young children: The ordering principle. Child Development, $\underline{48}$, 427-434.

Butterworth, B. (1999). The mathematical brain. London Eng.: MacMillan.

Cordes, S., Gelman, R., Gallistel, C. R., \& Whalen, J. (2000). Counting while talking: New evidence for nonverbal counting in human adults. UCLA, Manuscript under review.

Crump, T. ( 1990). The anthropology of numbers. Cambridge, Eng.: Cambridge Univ. Press.

Simon, T. (1999). Numerical thinking in a brain without numbers? Trends in Cognitive Science, $\underline{3}, 363-364$.

Dantzig, T. (1967). Number, the language of science. New York: The New York Free Press.

Dehaene, S. (1997). The number sense. Oxford, Eng.: Oxford Univ. Press.

Dehaene, S. \& Cohen, L. (1991). Two mental calculation systems: A case study of severe acalculia with preserved approximation. Neuropsychologia, 29, 1045-1074.

Dehaene, S., Spelke, E. S., Pinel, P., Stanescu, R., \& Tsivkin, S. (1999). Sources of mathematical thinking: Behavior and brain-imaging evidence. Science, 284, 970-97 4. 
Gelman \& Cordes

Droz, R. (1992). The multiple roots of natural numbers and their multiple interpretations. In J. Bideau, C. Mejac, \& J-P. Fischer (Eds.). Pathways to number: Children's developing numerical abilities. Hillsdale, NJ: Lawrence Erlbaum Associates (pp 229-244).

Evans, D. W. \& Gelman, R. (1982). Understanding infinity: A beginning inquiry. Unpublished manuscript, University of Pennsylvania, Philadelphia.

Fischer, J. P. (1992). Subitizing: The discontinuity after three. In J. Bideau, C. Mejac, \& J-P. Fischer (Eds.). Pathways to number: Children's developing numerical abilities. Hillsdale, NJ: Lawrence Erlbaum Associates, (pp 191-208).

Fuson, K. C. (1988). Children's counting and concepts of number. New York, SpringerVerlag.

Gallistel, C. R. (1990). The organization of learning. Cambridge, MA: The MIT Press.

Gallistel, C. R., Gelman, R., \& Cordes, S. (2000). The Cultural and Evolutionary History of the Real Numbers. In edited Fyssen volume based on the November, 1999, conference on Culture and Evolution.

Gallistel, C. R., \& Gelman, R. (1992). Preverbal and verbal counting and computation. Cognition, 44, 43-74.

Gelman, R. (1972). Logical capacity of very young children: number invariance rules. Child Development, $\underline{43}, 75-90$.

Gelman, R. \& Gallistel, C. R. (1978). The child's understanding of number. Cambridge, MA: Harvard U. Press.

Gelman, R. \& Greeno, J. G. (1989). On the nature of competence: Principles for understanding in a domain. Knowing and learning: Essays in honor of Robert Glaser. L. B. Resnick. Hillsdale, NJ, Erlbaum: 125-186.

Gelman, R. and Meck, E. (1983). Preschoolers' counting: Principles before skill. Cognition 13: 343-359. 
Gelman \& Cordes

Gvozdanoviâc, J. (1999). Numeral types and changes worldwide. Berlin; New York: Mouton de Gruyter.

Hartnett, P. M., \& Gelman, R. (1998). Early understandings of number: paths or barriers to the construction of new understandings? Learning and Instruction: The Journal of the European Association for Research in Learning and Instruction, $\underline{8}, 341-374$.

Holyoak, K. J. (1978). Comparative judgments with numerical reference points. Cognitive Psychology, 10, 203-243.

Holyoak, K. J. and Mah, W. A. (1982). Cognitive reference points in judgments of symbolic magnitudes.Cognitive Psycholology, 14, 328-352.

Ifrah, G. (1985). From one to zero. New York,: Viking.

McCleish, J. (1991). Number; The history of numbers and how they shape our lives. Fawcett Columbine: NYC, NY.

Mehler, J. \& Bever, T. G. (1967). Cognitive capacity of very young children. Science, $\underline{158}, 141-142$.

Moyer, R. S. \& Landauer, T. K. (1967). Time required for judgments of numerical inequality. Nature 215, 1519-1520.

Moyer, R. S. \& Landauer, T. K. (1973). Determinants of reaction time for digit inequality judgments. Bulletin of the Psychonomics Society. 1, 167-168.

Piaget, (1952). The child's understanding of number. Norton: New York.

Sophian, C. (1995). Children's numbers. Brown \& Benchmark: Madison, Wisconsin.

Trick, L. M. \& Pylyshyn, Z. (1993). What enumeration studies can showus about spatial attention: evidence for limited capacity preattentive processes. Journal of Experimental Psychology: Human Perception and Performance. 19, 331-51.

Uller, C., Carey, S., Huntley-Fenner, G., \& Klatt, L. (1999). What representations might underlie infant numerical knowledge? Cognitive Development, 13, 1-43.

Whalen, J., Gallistel, C. R., \& Gelman, R. (1999). Psychological Science.

Wynn, K. (1990). Children's understanding of counting. Cognition, 36, 155-193. 
Gelman \& Cordes

Zaslavsky, C. (1973). Africa counts. Boston, Prindle, Wever \& Schmidt. 
Gelman \& Cordes

\section{Figure Captions}

Figure 1. The counting principles and their relation to the arithmetic operations of addition, subtraction, and ordering (From Gelman \& Gallistel, 1978).

Figure 2. The Accumulator Model. A magnitude representing a numerosity is formed through accumulation of 'cup fulls' of activation, whereby each 'cup' represents one item, producing a total magnitude. Note that each discrete "next pour" in the process picks out the next magnitude. Accumulated magnitudes may be compared to a magnitude generated from memory and/or mapped to verbal/symbolic numerals for quantities. However, magnitudes read from memory have inherent scalar variability and so different retrievals will produce different magnitudes according to its probability density function. The greater the magnitude, the more likely an error.

Figure 3. (From Platt and Johnson, 1971.) A. The distribution in the number of presses rats make when attempting to approximate a given target number $\mathrm{N}(\mathrm{N}=4,8,16,24)$; $\mathrm{B}$. replot of the data to illustrate the signature of scalar variability.

Figure 4. The distributions in the number of presses adult humans make when attempting to rapidly press a key a given target number of times $(\mathrm{N}=2,3,4,5,8,13,20,32)$, without counting, while stating "the" coincident with every key press. Data are from the 4 subjects in Cordes, Gelman, Gallistel, \& Whalen (under review )who were run on the set sizes of 2-5.) 
Gelman \& Cordes

Acknowledgement

Work for this paper was partially supported by NSF grants DFS-9209741 and SRB-

97209741 to R.G. We Emmanuel Dupoux, C.R. Gallistel, Beth Levin, Dana Thadani and Osnat Zur for help comments on earlier versions . 